\title{
A Hybrid Approach for Enriching Image using Mamdani Neuro- Fuzzy Technique and its Comparative Analysis
}

\author{
Ashish Kumar Pandey \\ Master of Technology Scholar \\ Department of CSE \\ Integral University, Lucknow
}

\author{
Kavita Agarwal \\ Associate Professor \\ Department of CSE \\ Integral University, Lucknow
}

\author{
Mohd. Haroon \\ Associate Professor \\ Department of CSE \\ Integral University, Lucknow
}

\begin{abstract}
Surveys have proven that the including images in a content to complement the text amplify the likelihood of people reading and engaging with your article. The straightforward reason is this that since people are naturally attracted to and tempted by pictures. Its established by a research work that whenever we include images in an article it increases their view approximately $94 \%$ more views, and the same is true for press releases, with an increase in views of $45 \%$. Thus the right image makes a huge difference in the overall impression and effectiveness of your website. Hence the idea and concept of image enhancement has a important role in today's life. Image enhancement as defined is a concept of enriching the perception of images for human viewers. The main objective is to reduce impulsive noise, sharpen the edges, and improvise the general appearance with the help of different image enhancement techniques. Many techniques have been successfully implemented which include contrast enhancement using histogram equalization techniques. It enhances the contrast of images by transforming the values in an intensity images. Soft computing techniques like Fuzzy technique can supervise the ambiguity and imperfection of an image which can be represented in the form of fuzzy sets. Spatial domain method which can operate directly on the pixels values. In this research work we get in a hybrid approach of mamdani based Neuro Fuzzy technique for enriching image quality. The Mamdani Neuro Fuzzy inference system shows its advantage in output expression and thus it is used in this project. Also a comparative analysis is done for image enhancement using histogram equalization, Fuzzy techniques, spatial domain method and neuro fuzzy technique. This is based on the RMSE value of the images. To implement this research work we used MATLAB 7.8.0(R2009a) and NeuCom Tools.
\end{abstract}

\section{Keywords}

Mamdani Neuro Fuzzy System, Fuzzy logic, Histogram Equalization Technique, Spatial domain method.

\section{INTRODUCTION}

Great difference images talking itself and protecting details. Such images are needed for some imperative regions to be specific machine vision, remote sensing, dynamic and activity scene examination, biomedical image investigation and selfgoverning route. However a huge bit of the recorded pictures encounter the evil impacts of poor distinction due to the lacking lighting in the midst of image acquiring, inaccurately setting of opening size and screen speed furthermore nonlinear image intensities mapping. Thusly we get an image with poor separation. Thus it is important to enhance the image quality utilizing certain sort of image enhancement techniques. The procedure by which we enhance the quality of the image is known as image enhancement. Hence, image enhancement is being utilized to build the nature of the image. Image enhancement is a fundamental task connected in image preparing to enhance interpretability and appearance of the image. It gives better include image to further image preparing assignment.

Image enhancement is defined by two dimensional function that is $\mathrm{g}(\mathrm{x}, \mathrm{y})=\mathrm{T}[\mathrm{f}(\mathrm{x}, \mathrm{y})]$. Where $\mathrm{T}$ is the transformation function. The Lena image (Image 1) is most popular image because many researchers is used this image for their research and give the different results with different- 2 parameters. We also used this image to compare the existing techniques and proposed MNFIE and also compare to the existing (Lena image) results based on the RMSE value. And we used one more new image i.e. Haroon image (Image2) to compare the existing image techniques and the proposed MNFIE and find out the proportional results. This image is not used by any researchers.

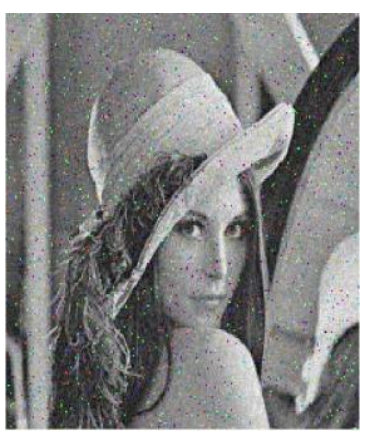

Image 1(Lena)

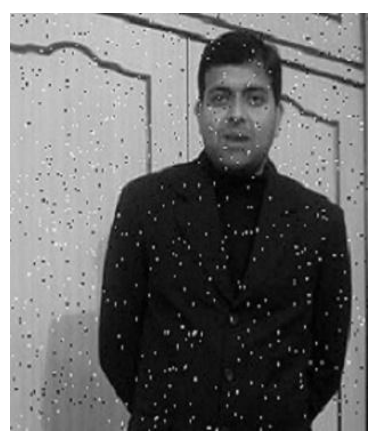

Image 2(Haroon)
There are many existing techniques available for image enhancement. Like contrast enhancement technique, spatial domain techniques, frequency domain techniques, soft computing based techniques [11, \&12]. This research works concentrates on the soft computing based image augmentation techniques, contrast enhancement technique and spatial domain technique. Over the years there have been successful implementation of fuzzy based image enhancement techniques, neural network based image enhancement techniques and neuro fuzzy image enhancement techniques. Each of the techniques has their own advantages and disadvantages. All of the neuro fuzzy image enhancement techniques have been apply using ANFIS model and are based on Takagi Sugeno model. It is seen that Mamdani based fuzzy system is more human understandable hence we, propose a neuro fuzzy technique of image enrichment based on mamdani based neuro fuzzy system. 


\section{LITERATURE SURVEY}

In the field of image noise reduction, several linear and nonlinear filtering methods have been planned. Linear filters don't effectively eliminate impulse noise as they have a tendency to blur the edges of an image. Nonlinear filters like median filters are better suited for dealing with impulse noise. There are some fuzzy based nonlinear filters. The fuzzy median filter is a modification to the classical median filter. The Fuzzy Inference Rules by Else action (FIRE) filters are a family of non-linear operators that adopt fuzzy rules to remove noise from images. Russo introduced a multi-pass fuzzy filter consisting of three cascade blocks in. Khriji and Gabbouj developed a multi-channel filter by combine fuzzy rational and median functions. These filters conserve the edges and chromaticity of the image [9, 11]. Wenbin accessible a novel idea of alpha trimmed mean and the similarity of pixels for the detection of impulse noise based on a large difference between the noisy pixel and the noise free pixel. Rank -order mean (ROM) is a hybrid filter with capability to devise a Least-Mean Square Design for filtering out the fixed and non- linear desire noise. Efforts are made in to reduce blurring at the edges due to linear filtering. A signal adaptive median filtering algorithm is proposed in for the removal of impulse noise in which the notion of homogeneity level is defined for pixel values based on their global and local statistical properties. Median based filters are modified for detail safeguarding Mansoor developed a recursive filter images which are highly corrupted by impulse noise $[1,5,7$, \&12]. This filter estimates the noise level which is required to obtain the filter parameters. All these filters were specifically designed to reduce impulse noise. R.Pushpavalli and G.Sivarajde proposed Sugeno based Neurofuzzy image improvement technique using tractate median filter.

\section{BACKGROUND}

Neuro-fuzzy system is a fuzzy system that uses a taking in calculation got from or motivated by neural network theory to focus its parameters (fuzzy sets and fuzzy rules) by preparing information tests. Neuro-fuzzy was proposed by J. S. R. Jang. Neuro Fuzzy System synergizes these two techniques by combining the human-like reasoning style of fuzzy systems with the learning and connectionist structure of neural networks $[7,9,13]$.

\section{TYPES OF NEURO FUZZY SYSTEM}

- Mamdani based neuro Fuzzy inference system.

- Takagi Sugeno fuzzy inference system

Mamdani fuzzy inference systems have following advantages:

- It's intuitive.

- It has widespread acceptance.

- It's well suited to human cognition.

Takagi Sugeno fuzzy inference system guarantee continuity of the output surface but it has difficulties in dealing with the multi-parameter synthetic assessment; it has difficulties in assigning weight to each input and fuzzy rules.

ANFIS implements only Takagi sugeno based Fuzzy inference system [12]. The Mamdani fuzzy inference system shows its advantage in output expression and thus it is used in this project.

\section{EXISTING METHODOLOGY AND DRAWBACKS}

- Contrast Enhancement: It make over the pixels values in to an intensity images by which the contrast of the images increased.

- Spatial domain methods: directly deal with the image pixels. The pixel values are manipulated to achieve desired enhancement

- $\quad$ Frequency domain methods: based on the manipulation of the orthogonal transform of the image rather than the image itself.

- Fuzzy domain: Gray level mapping into a fuzzy plane, membership transformation function, allows imprecise/contradictory inputs

- $\quad$ Neuro Fuzzy ANFIS based method (Sugeno -type fuzzy systems): one input and one output, guarantees continuity of the output surface

\section{DRAWBACKS:}

The above image enhancement techniques have some advantages and disadvantages. Some of the noted disadvantages are:

- Spatial and frequency come near: Time unbearable.

- Contrast Enhancement: It enhanced the contrast of the images but sometimes images lacks brightness preservation.

- Fuzzy grayscale enhancement: Enhances low contrast images only.

- Sugeno based Neuro-fuzzy image enhancement technique: are complex and complicated for human interpretation $[5,22, \& 26]$.

- Linear filter: Tend to blur edges and other image detail.

\section{PROPOSED METHODOLOGY AND PROPOSED ALGORITHM}

In this method we use Mamdani based Neuro Fuzzy Model. The proposed work is carried in two stages:

- In first stage, noisy image is de-noised by Median filter. (Call subroutine of MNFIE)

- The de-noised image is further enhanced by MNFIE.

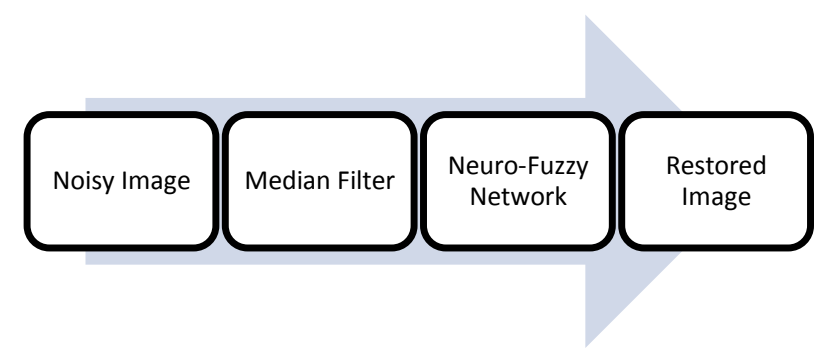

Figure 1 : Proposed MNFIE

Median filter (non-linear filter) which uses the median value to replace the corrupted pixel, and these filters have the capability to remove impulsive noise while preserving the edges. Hence we go for median filter.

\section{ALGORITHM 1: WORKING OF MNFIE SYSTEM}

Step1: Select a poor quality of image or an image with noise and read it using imread() function.

Step2: If the selected image is in RGB then converted to gray scale format using rgb2gray() function.

Step3:To the non-linear filter, salt and pepper noise is used to 
made noisy image.

Step4: The image which is find out after the filter can be considered as an original image.

Step5: The Enhanced image can be obtained by differentdifferent enhancement technique for the original image.

Step6: Call the subroutine of Mamdani Neuro-fuzzy image enhancement (MNFIE).

Step7: The enhanced image find-out in step5 is compared with MNFIE enhanced image on the basis of root mean square error value. If obtained RMSE value is lesser that means the quality of an image is good.

\section{ALGORITHM2: SUBROUTINE OF MNFIE SYSTEM}

Step1: The filtered image pixel values are stored in .csv file and are loaded to the neuro-fuzzy tools neucom.

Step2: It is measured as training images and are obtained by corrupting the original training image by impulse noise of $50 \%$ noise density.

Step3: Mamdaniv Neuro - fuzzy network are provides iterative optimization for their parameter so that its output converges to unique noise free image.

Step 4: When we load the data in neuro fuzzy tool for the training its provide the least error and is made fixed for testing the image.

Step 5: Images are tested.

Step 6: Return output.

\section{IMPLEMENTATION}

Neuro-fuzzy system is a fuzzy system that uses a taking in calculation got from or motivated by neural network theory to focus its parameters (fuzzy sets and fuzzy rules) by preparing information tests. On the premise of taking after a image enhancement calculation has been developed and implemented. The Lena image (Image 1) is most accepted image since several researchers is used this image for their research and give the different-2 results with different-2 parameters. We also used this image to compare the existing techniques and proposed MNFIE and also evaluate to the existing (Lena image) results based on the RMSE value. And we used one more new image i.e. Haroon image (Image2) to compare the existing image techniques and the proposed MNFIE and find out the comparative results. This image is not used by any researchers.

\section{For Lena image:}

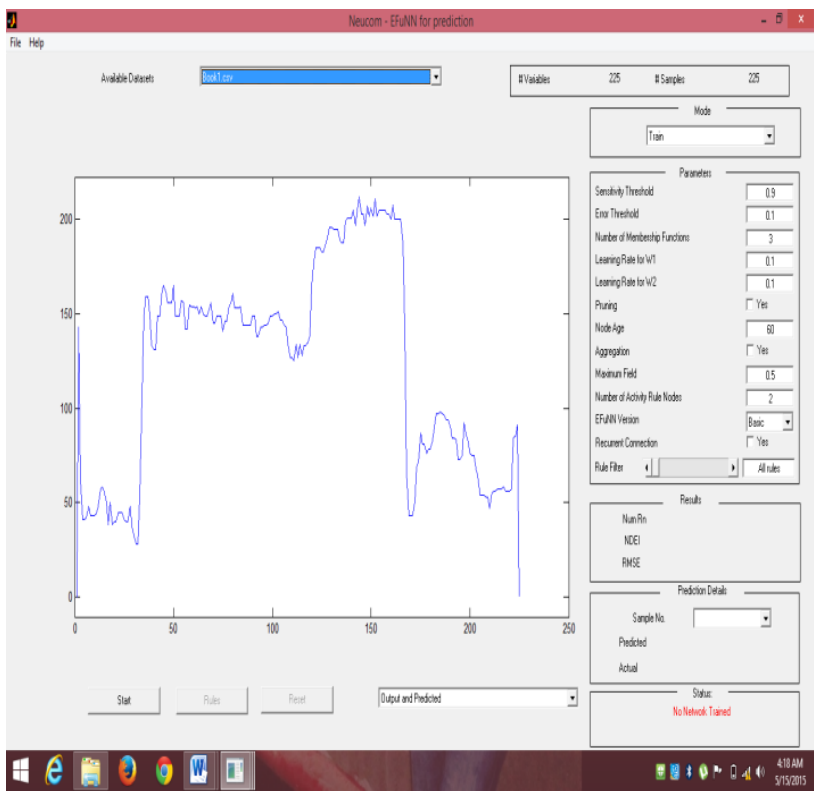

Figure 2: Data of image 1 in .CSV format loaded to EFuNN

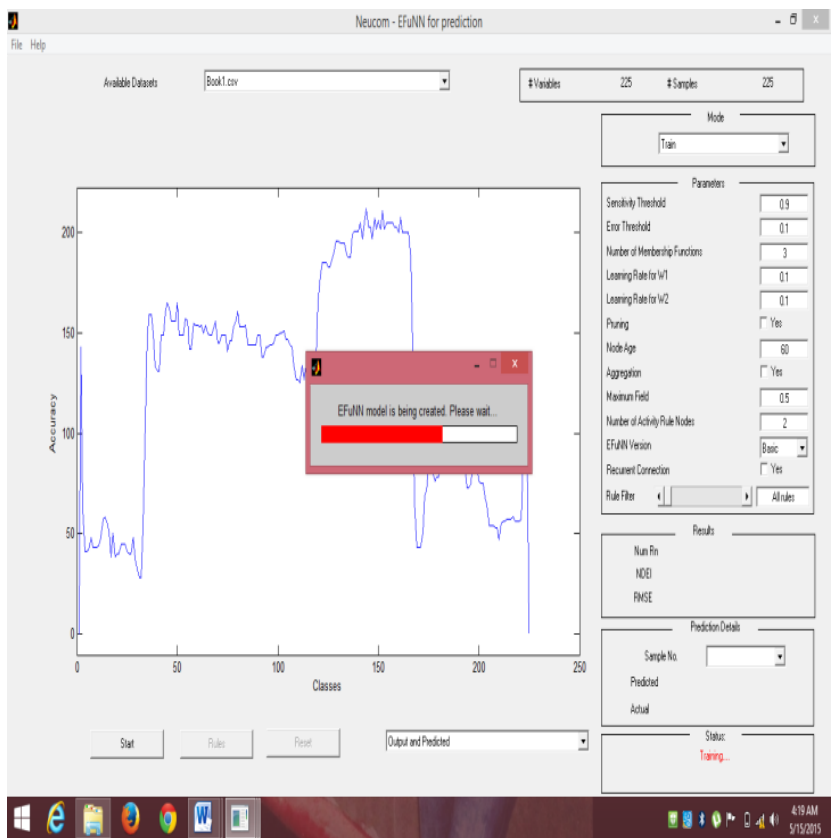

Figure 3 : Training of EFuNN 


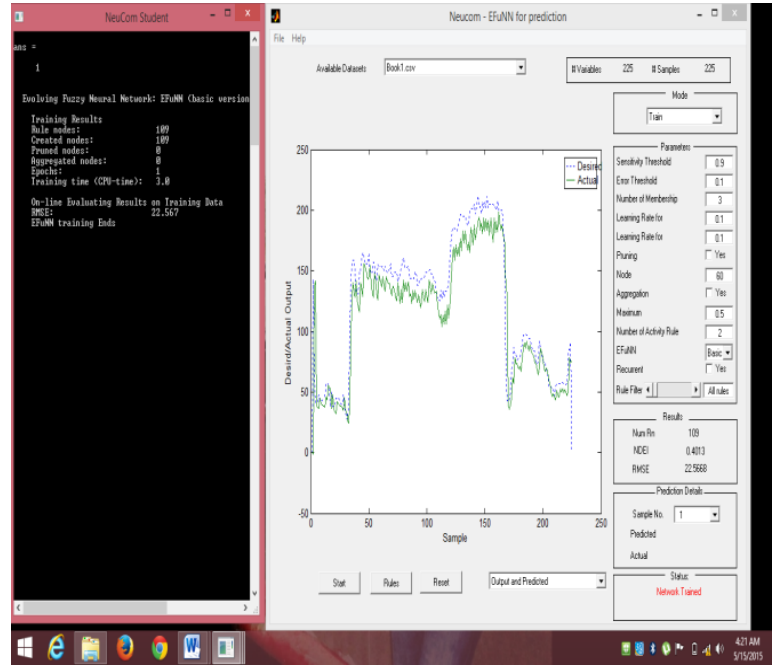

Figure 4 : Training outcome of EFuNN

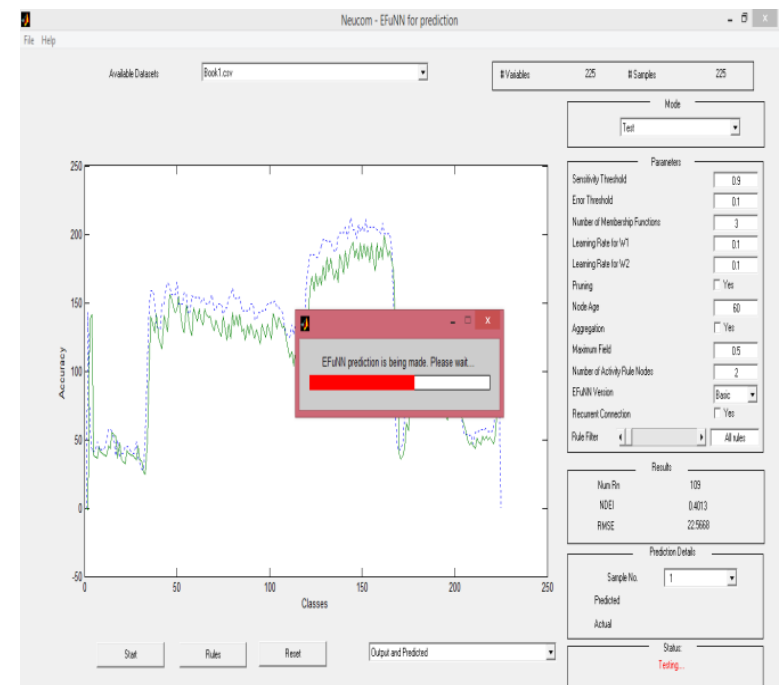

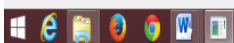

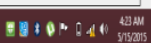

Figure 5 : Testing EFuNN

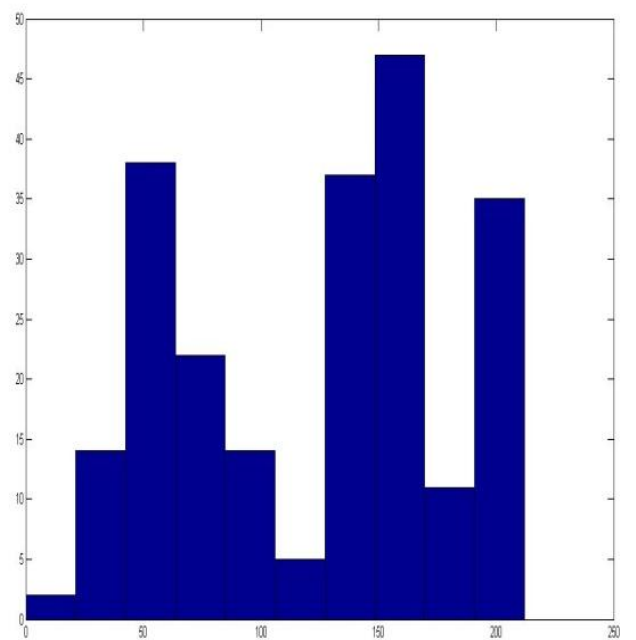

Figure 6 : Histogram for Actual Pixel value

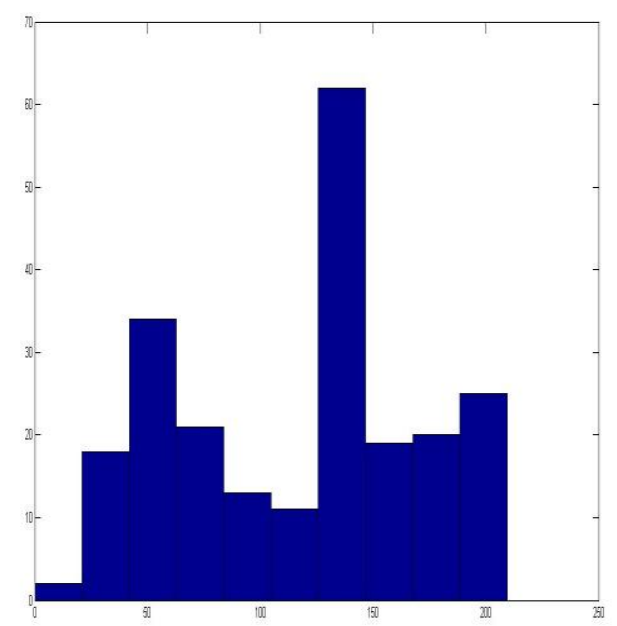

Figure 7 : Histogram for Predicted Pixel value

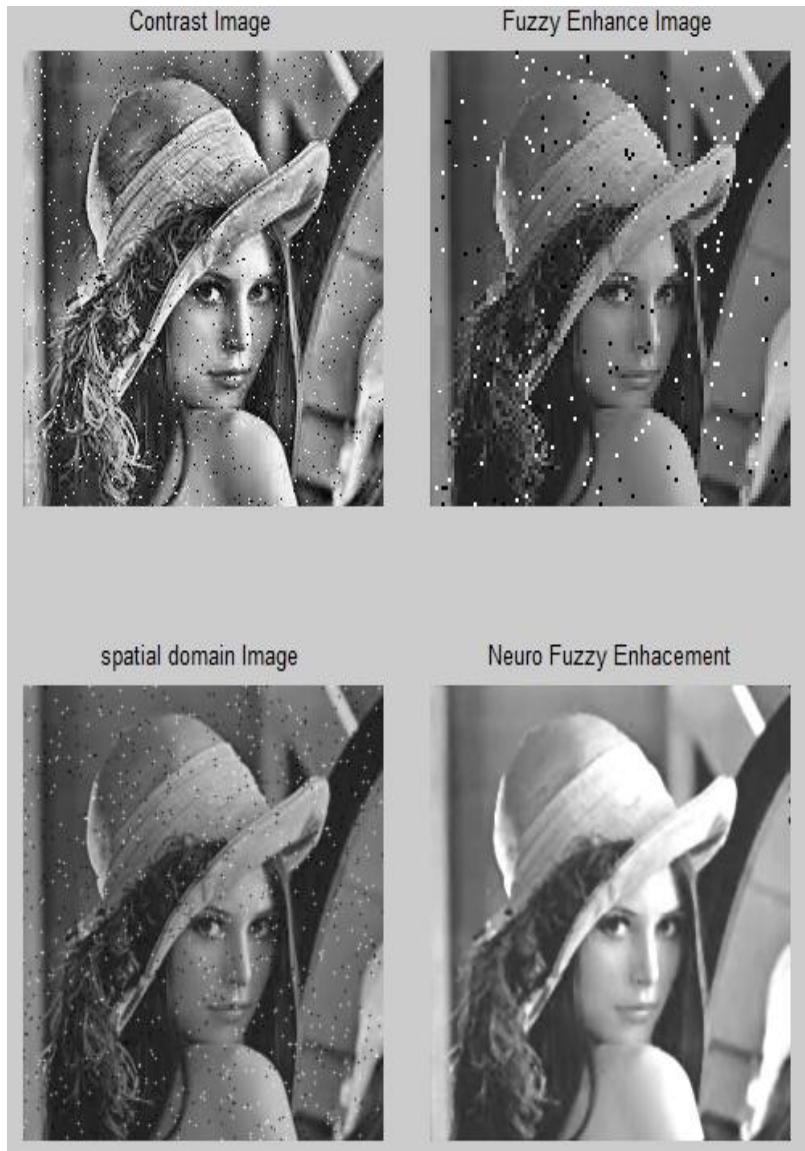

Figure 8 : Final output of Image enhancement from MNFIE

\section{RESULT 1 FOR LENA IMAGE}

We write a code in MATLAB to implement the algorithm and also find-out the results. 
Table 1 : Comparative Results for lena Image based on RMSE

\begin{tabular}{|c|c|c|c|}
\hline Sr.No. & Enhancement Technique & Existing RMSE Value & Proposed RMSE value \\
\hline 1 & Contrast Enhancement & 10.284 & 9.102 \\
\hline 2 & Fuzzy Enhancement & 11.6552 & 8.012 \\
\hline 3 & Spatial Domain Method & 10.138 & 9.012 \\
\hline 4 & ANFIS/MNFIE & 11.7547 & 5.1021 \\
\hline
\end{tabular}

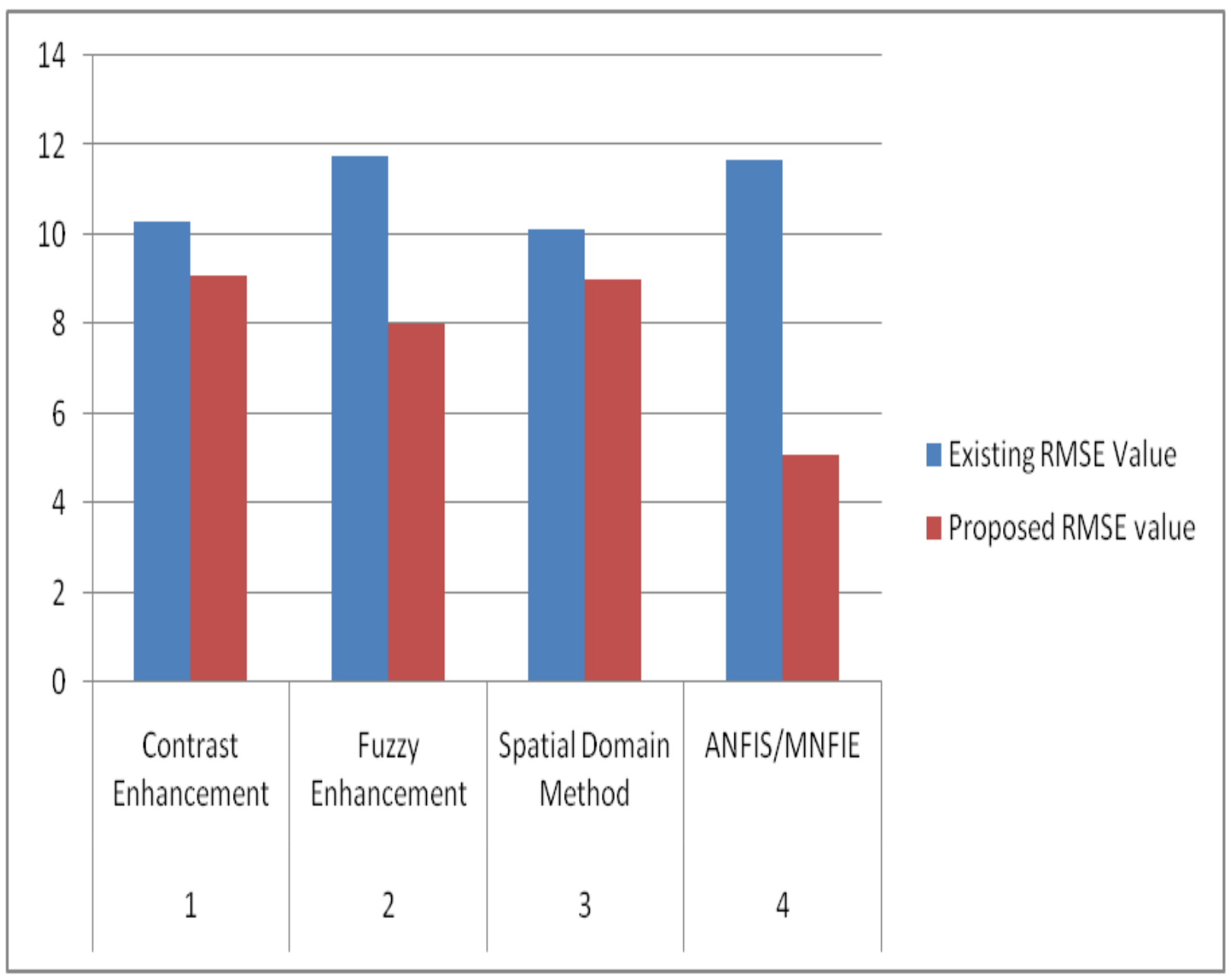

Figure 9 : Performance of RMSE for proposed algorithm and Existing RMSE value 
For Haroon Image

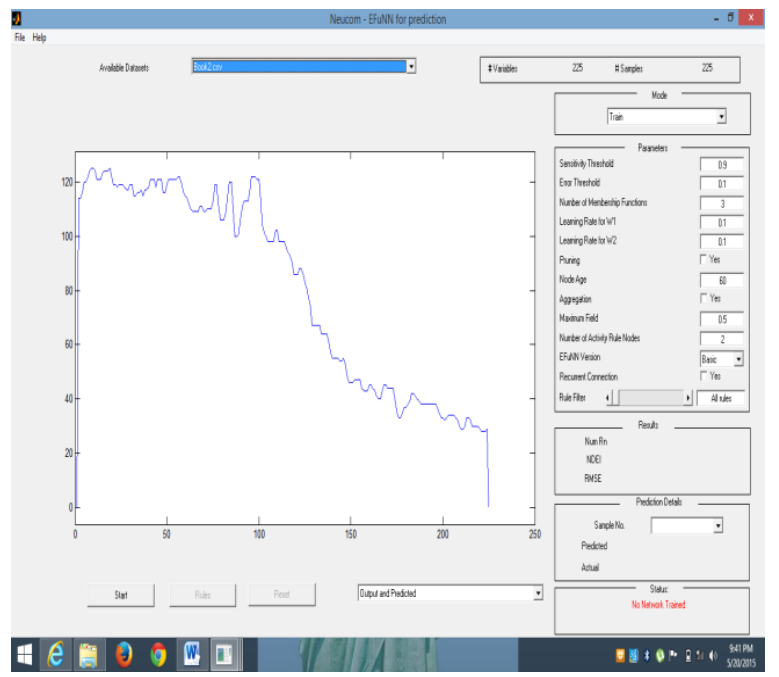

Figure 10 : Data of image2 in .CSV format loaded to EFuNN
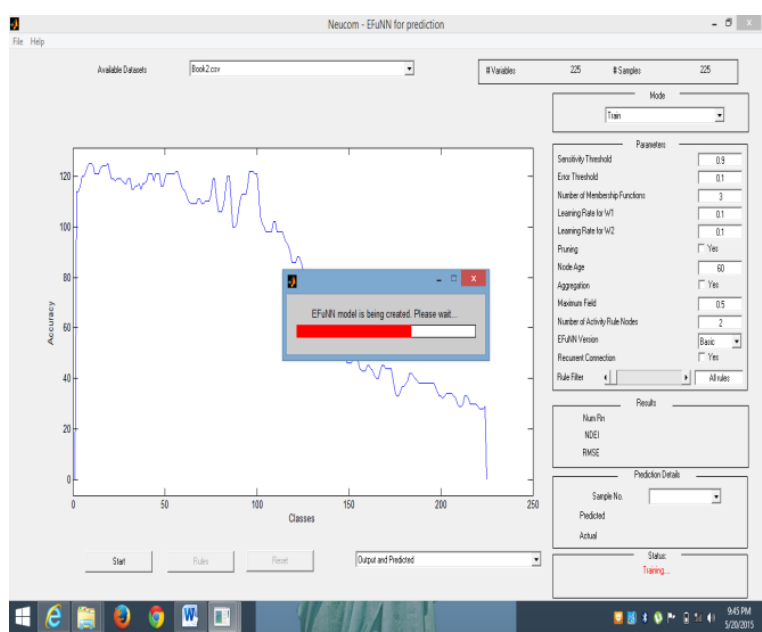

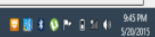

Figure 11 : Training of EFuNN

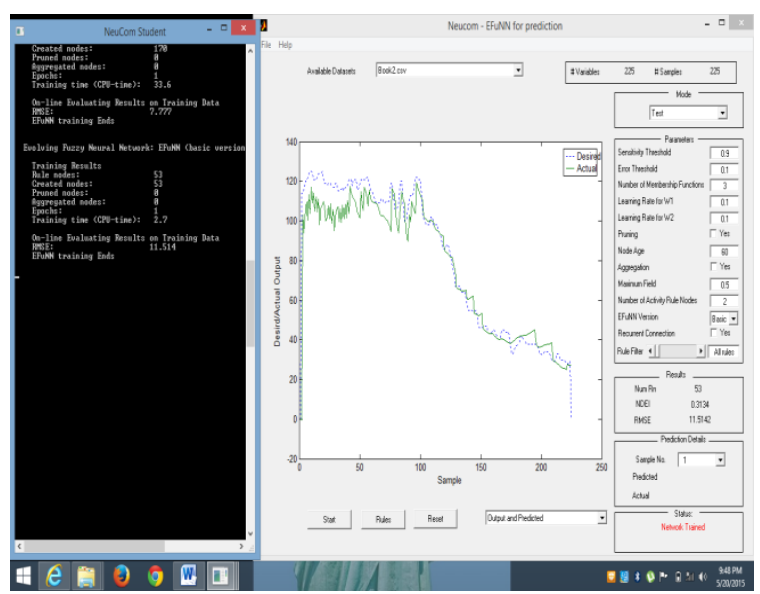

Figure 12 : Training outcome of EFuNN

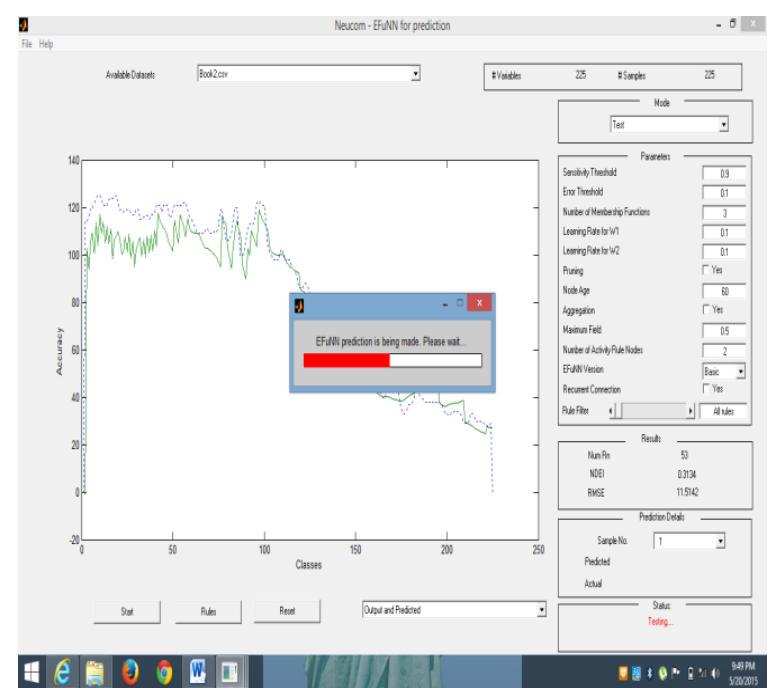

Figure 13 : Testing of EFuNN
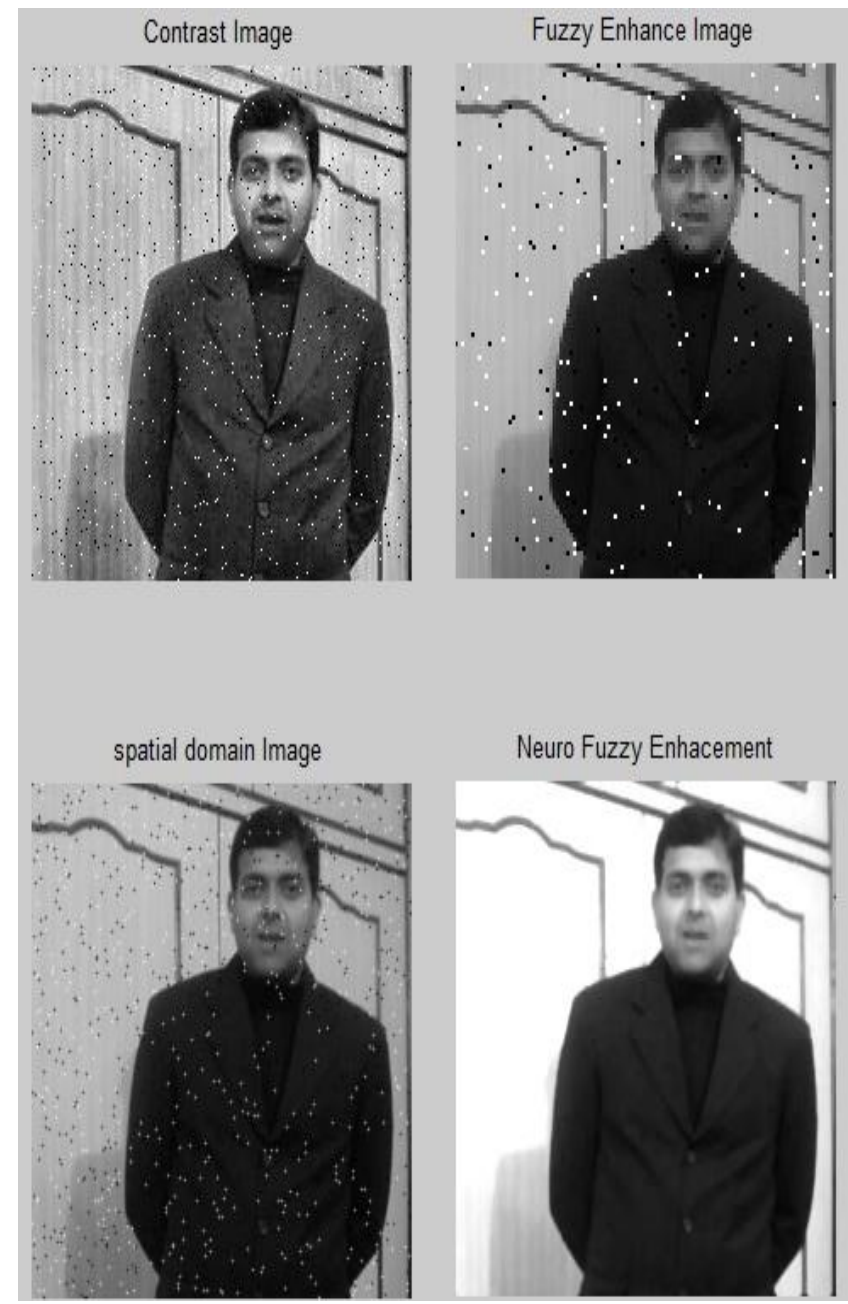

Figure 14 : Final output of Image enhancement from MNFIE 


\section{RESULT 2 FOR HAROON IMAGE}

Table 2 : Proposed Algorithm Results for Haroon Image

\begin{tabular}{|c|c|c|}
\hline Sr.No. & Enhancement Technique & RMSE Value \\
\hline 1 & Contrast Enhancement & 9.0259 \\
\hline 2 & Fuzzy Enhancement & 9.1065 \\
\hline 3 & Spatial Domain Method & 5.0954 \\
\hline 4 & The Proposed MNFIE & \\
\hline
\end{tabular}

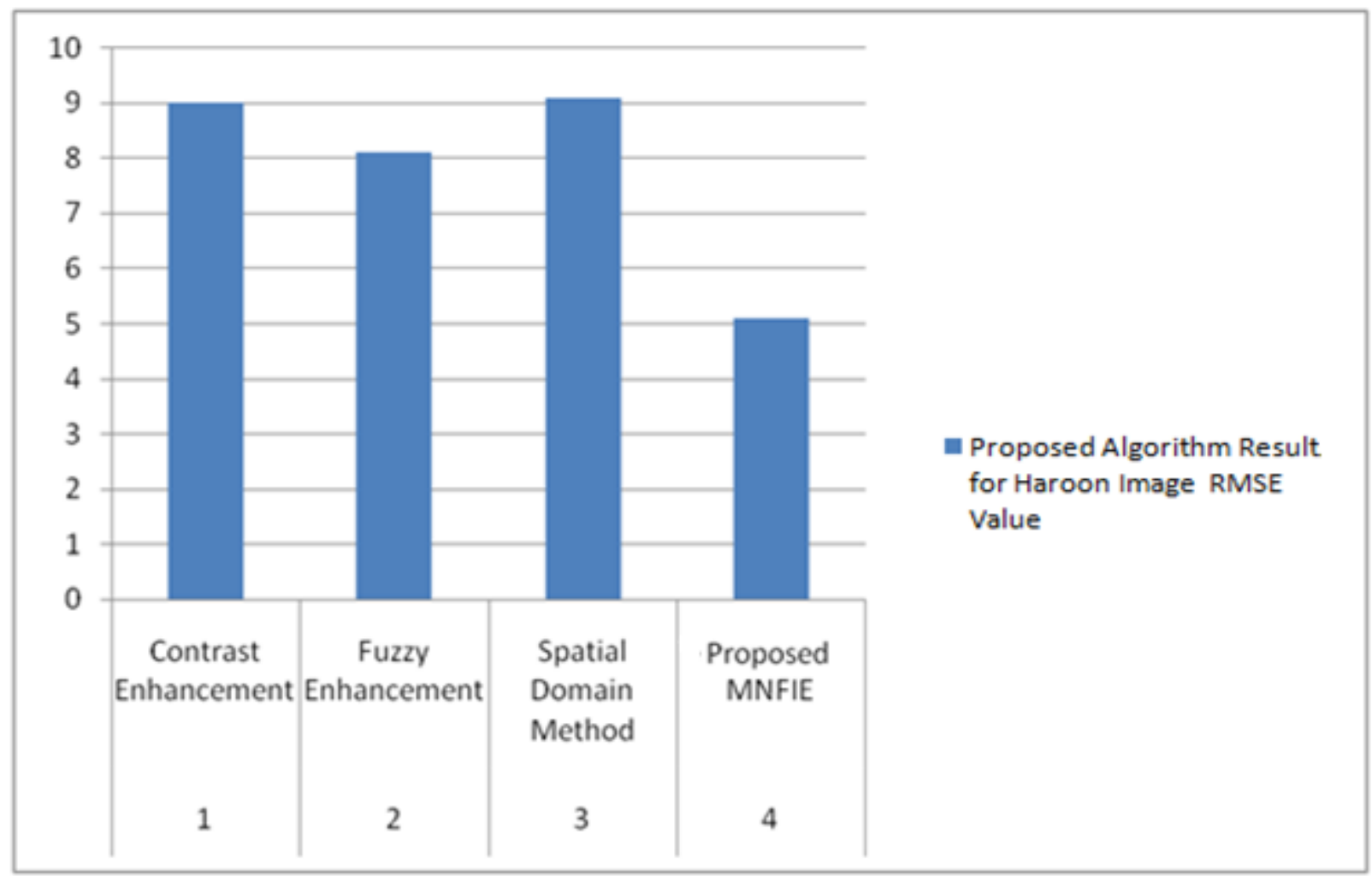

Figure 15: Performance of RMSE for proposed algorithm

On the basis of the results the proposed MFFIE is better than to existing image enhancement techniques.

\section{CONCLUSION AND FUTURE WORK}

There are many image enhancement techniques but most of them are computational. Soft computing has offered wide scope of new methods of computing. In this research work a new image enhancement method using neuro fuzzy method was proposed. It used mamdani based fuzzy inference system. A noisy image was selected and the image was enhanced using normal histogram image enhancement method, fuzzy image enhancement method, spatial domain method and proposed mamdani based neuro fuzzy image enhancement method. The implementation was carried out in MATLAB7.8.0 (R2009a) and Neucom. After the testing and results we find-out the proposed method is far better contrast enhancement method than the said ones. The implementation is done on the basis of root mean square error. Lower the RMSE value means better the image quality.

As a future work the image enhancement can be carried out for noisy images with high noise ratios and blurred images using other filter and proposed MNFIE methods.

\section{REFERENCES}

[1] Amaninder Kaur Brar, Vikas Wasson (2014), "Image Denoising Using Improved Neuro-Fuzzy Based Algorithm: A Review ”, vol. 4 Issue 4, International Journal of Advanced Research in Computer Science and Software Engineering ,ISSN:2277 128X.

[2] K. Moniga, S.ArunKumar (2014),“ Evaluation of Various Edge Detection Algorithms - Review”, INTERNATIONAL JOURNAL OF ENGINEERING SCIENCES \& RESEARCH TECHNOLOGY,ISSN: 
2277-9655, Scientific Journal Impact Factor: 3.449 (ISRA), Impact Factor: 2.114,pp.331-337.

[3] D. Ezhilmaran , M. Adhiyaman (2014),“ A Review Study on Fingerprint Image Enhancement Techniques", International Journal of Computer Science \& Engineering Technology (IJCSET) ISSN:22293345,Vol.5, Issue 6,pp.625-631.

[4] Anant Bhardwaj,Kapil Kumar Siddhu (2013), "An Approach to Medical Image Classification Using Neuro Fuzzy Logic and ANFIS Classifier", vol. 4 Issue3, International Journal of Computer Trends and Technology,ISSN:2231-2803.

[5] R.Pushpavalli,G.Sivarajde (2013), "Image Enhancement Using Adaptive Neuro-Fuzzy Inference System ”, vol. 2 Issue 6, International Journal of Scientific \& Technology Research,ISSN:2277-8616.

[6] Ahmed F. and Das S. (2013) "Removal of High Density Salt and Pepper Noise in Images with an Iterative Adaptive Fuzzy Filter using alpha-trimmed Mean" IEEE Transactions on Fuzzy Systems.

[7] Er.Mandeep Kaur, Er.Kiran Jain,Er. Virendra Lather (2013),“ Study of Image Enhancement Techniques: A Review", International Journal of Advanced Research in Computer Science and Software Engineering, ISSN: 2277 128X, Vol.3,Issue 4, pp. 846-848.

[8] S.S.Bedi,Rati Khandelwal (2013),“ Various Image Enhancement Techniques- A Critical Review", International Journal of Advanced Research in Computer and Communication Engineering ,ISSN(Print):23194940 ,ISSN(Online):2278-1021,Vol. 2, Issue 3,pp.16051609.

[9] Mohammad Haroon, Mohd Husain,“ Interest Attentive Dynamic Load Balancing in Distributed Systems ", Computing for Sustainable Global Development (INDIACom), 2015 2nd International Conference on , 1116 - 1120, ISBN:978-9-3805-4415-1, 11-13 March 2015 .

[10] Shina Dhingra, Palvinder Singh Mann (2013),“ An Adaptive Neuro Fuzzy Approach for Software
Development Time Estimation", International Journal of Advanced Research in Computer Science and Software Engineering ,ISSN: 2277 128X,Vol.3, Issue 5,pp.586590.

[11] Manish Madhav Tripathi, Mohd Haroon, Minsa Jafar, Mansi Jain,“ Maxillofacial Surgery Using X-Ray Based Face Recognition by Elastic Bunch Graph Matching", springer Berlin Heidelberg, 2010/1/1.

[12] Manish Madhav Tripathi, Mohd Haroon, Minsa Jafar, Mansi Jain, "Contemporary Computing: Second International Conference, IC3 2010, Noida, India, August 9-11, 2010. Proceedings ", Springer Science \& Business Media, 2010/7/23.

[13] L.I. Kuncheva, F. Steimann (1999), "Fuzzy diagnosis," Artificial Intelligence in Medicine, vol. 16, pp. 121-128.

[14] D. Ghimire and J. Lee (2011),“ Nonlinear Transfer Function-Based Local Approach for Color Image Enhancement," IEEE Transactions on Consumer Electronics, Vol. 57, No. 2.

[15] H. Zhang, Q. Zhao, Lu Li, Y.c. Li, Y.h. You (2011),“ Muti-scale Image Enhancement Based on Properties of Human Visual System," 4th International Congress on Image and Signal Processing.

[16] Snehal O.Mundhada , Prof. V. K. Shandilya (2012),“ Image Enhancement using a Combined Approach of Spatial and Transformation Domain Techniques", International Journal of Emerging Research in Management \&Technology,ISSN: 2278-9359, ,pp.1-4.

[17] Nirmala S. O, T. D. Dongale, R. K. Kamat (2012),“ Review on Image Enhancement Techniques: FPGA Implementation perspective", International Journal of Electronics Communication and Computer Technology (IJECCT),ISSN: 2249-7838,Vol.2, Issue 6,pp.270-274.

[18] Nirmala S. O, T. D. Dongale, R. K. Kamat (2012),“ Review on Image Enhancement Techniques: FPGA Implementation perspective", International Journal of Electronics Communication and Computer Technology (IJECCT), ISSN: 2249-7838,Vol.2, Issue 6,pp.270-274. 